\title{
Infants and Children with Stage 4 Neuroblastoma Express Significantly Different Levels of Specific Molecular Markers
}

Stefano Parodi ${ }^{1}$, Giovanni Erminio², Barbara Carlini ${ }^{3}$, Alberto Garaventa $^{4}$, Paolo D’Angelo $^{5}$, Riccardo Haupt ${ }^{2}$, and Maria Valeria Corrias ${ }^{3 *}$

${ }^{1}$ Clinical Epidemiology, IRCCS San Martino-IST, Largo Benzi 5, 16137 Genoa, Italy

${ }^{2}$ Epidemiology and Biostatistics Section, Scientific directorate, IRCCS Istituto Giannina Gaslini, Largo Gaslini 5, 16147 Genoa, Italy

${ }^{3}$ Laboratory of Oncology, and Department of Hematology-Oncology, IRCCS Istituto Giannina Gaslini, Largo Gaslini 5, 16147 Genoa, Italy

${ }^{4}$ Department of Hematology-Oncology, IRCCS Istituto Giannina Gaslini, Largo Gaslini 5, 16147 Genoa, Italy

${ }^{5}$ Ospedale dei Bambini, Via Delle Benedettine 1, 90134 Palermo, Italy

\begin{abstract}
In neuroblastoma (NB), age at diagnosis is a strong prognostic factor. Hereby, we evaluated whether patients with metastatic NB below 1 year of age (infants) expressed lower levels of NB-specific molecular markers compared to patients over 1 year (children). Bone marrow (BM) and peripheral blood (PB) samples collected at diagnosis from 54 Italian patients with metastatic NB were analysed by RT-qPCR for $T H, P H O X 2 B$ and DCX mRNA expression. Statistical analyses were then performed to evaluate the diagnostic performance and the association with other known prognostic factors.

In spite of similar levels of morphological BM infiltration, $T H, P H O X 2 B$ and $D C X$ expression levels in $B M$ and $P B$ samples were significantly lower in stage 4 infants than in children. Between infants with stage 4 and $4 S$, the former expressed significantly higher levels in BM samples but similar levels in PB samples. In children with stage 4 NB, TH expression levels significantly associated with the presence of BM and skeletal metastasis.

Thus, RT-qPCR analysis of BM and PB samples showed potential clinical significance that should be evaluated in future multicentre prospective studies for infants with metastatic NB in comparison with conventional morphological evaluation and other prognostic factors evaluable in the primary tumors.
\end{abstract}

Keywords: Neuroblastoma; Gene expression; Metastasis; Tyrosine hydroxylase; Doublecortin; Paired-like homeobox 2b

Abbreviations: TH, tyrosine hydroxylase; DCX, Doublecortin; PHOX2B, Paired-like homeobox 2b.

\section{Introduction}

Neuroblastoma (NB) clinical presentation is highly heterogeneous. Metastatic spread is present in $50 \%$ of cases at diagnosis and mainly involves the bone marrow (BM). The main prognostic factors are stage (International Neuroblastoma Staging System (INSS) [1]), age at diagnosis, and $M Y C N$ oncogene status [2]. Being a continuous variable, the effect of age on prognosis is not discrete [2-4], but an age cut off at 12 months has been used for patient risk stratification until the International Neuroblastoma Risk Group Stratification System (INRG-SS) shifted the age cut off to 18 months [2].

Prognosis of stage 4 patients over 12 months of age (children) is dismal in spite of intensive multimodal therapies [2]. Conversely, prognosis of infants (<12 months) with either stage 4 or stage $4 S$ (S for special [5]), is very good [6,7], unless they present with $M Y C N$-amplified tumors $[6,8]$. However, while infants with stage $4 \mathrm{~S}$ disease receive no or little treatment, usually targeted to life-threatening symptoms [6], stage 4 infants require low dose chemotherapy to achieve cure [7]. Thus, children and infants with metastatic NB have remarkable different outcomes, following remarkable different therapeutic regimens.

The mechanisms responsible for such profound differences in relation to age at diagnosis have been ascribed to a higher frequency of favourable histology [7], and to more favourable genetic profiles in infants than in children [9-13]. Hereby, we investigated whether infants with stage 4 disease had levels of infiltration lower than stage 4 children. To test this hypothesis we used standardized RTquantitative(q)PCR [14] for TH, PHOX2B and DCX, in paired BM and peripheral blood (PB) samples collected at diagnosis. In these samples, tyrosine hydroxylase, the first enzyme of catecholamine synthesis; the neuronal-specific transcription factor, PHOX2B; and Doublecortin, a regulator of neuronal microtubule assembly; were all shown to be expressed only by the infiltrating neuroblastoma cells [15]. Since by INSS [1] definition infants with stage $4 \mathrm{~S} \mathrm{NB}$ must have lower BM infiltration than infants with stage 4 disease, these latter patients were considered as reference group.

\section{Materials and Methods}

\section{Patients and samples}

Between January 2001 and June 2008, three-hundred and twenty Italian NB patients were staged at diagnosis as 4 , fifty infants as 4 and sixty-four infants as 4S [1]. Demographic, clinical and follow-up data were retrieved from the Italian NB Registry (INBR) that collects data at diagnosis, during treatment and at least yearly after treatment discontinuation [16]. All patients/guardians signed a written consent allowing the use of samples and clinical data for research purposes. The study was approved by the Ethics Committees of each Italian centre and the procedures were in accordance with the Helsinki Declaration. Diagnostic and therapeutic details are available in Document S1.

*Corresponding author: Maria Valeria Corrias, Head Molecular Oncology Laboratory of Oncology, Gaslini Institute Largo Gaslini, 516147 Genoa Italy, Tel: +39 0105636524 Fax: +39 010 3779820; E-mail: mariavaleriacorrias@ospedale-gaslini.ge.it

Received February 21, 2012; Accepted March 29, 2012; Published April 05, 2012 Citation: Parodi S, Erminio G, Carlini B, Garaventa A, D'Angelo P, et al. (2012) Infants and Children with Stage 4 Neuroblastoma Express Significantly Different Levels of Specific Molecular Markers. J Mol Biomark Diagn S2:008. doi:10.4172/2155-9929.S2-008

Copyright: ( 2012 Parodi S, et al. This is an open-access article distributed unde the terms of the Creative Commons Attribution License, which permits unrestricted use, distribution, and reproduction in any medium, provided the original author and source are credited 
Only 18 paired $\mathrm{BM}$ and $\mathrm{PB}$ samples were available from stage 4 infants, thus to obtain three homogeneous groups we randomly selected 18 stage 4 children and 18 stage $4 \mathrm{~S}$ infants among those with available paired samples. Before starting the molecular analysis we checked whether the selected patients fully represented the entire cohort. As shown in Table S1, the selected patients were not significantly different from the excluded ones as for demographic, biological and clinical features, including outcome.

\section{Detection of NB-related markers in BM and PB samples}

Half $\mathrm{mL}$ of each $\mathrm{BM}$ aspirate and $2 \mathrm{~mL}$ of $\mathrm{PB}$, taken under sedation and diluted in Acid Citric-Dextrane anticoagulant, were shipped overnight at $4^{\circ} \mathrm{C}$ to the Italian reference laboratory. Total RNA was then extracted as previously described [17], and reverse transcribed according to standard operating procedures [14]. Five $\mu \mathrm{L}$ of cDNA in triplicate were then separately amplified for 40 cycles with the specific primers and probe for $\beta 2$-microglobulin (endogenous reference mRNA, forward primer: 5'-GAGTATGCCTGCCGTGTG-3'; reverse primer; 5'-AATCCAAATGCGGCATCT-3'; probe 5'-CCTCCATGATGCTGCTTACATGTCTC -3', TH (forward primer: 5'-ATTGCTGAGATCGCCTTCCA-3'; reverse primer; 5'-AATCTCCTCGGCGGTGTACTC-3'; probe5'-ACAGGCACGGCGACCCGATTC-3'[14], PHOX2B (forward primer: 5'-CAGGGACCACCAGAGCAGT-3'; reverse primer; 5- CTGCTTGCGCTTCTCGTTGA-3'; probe 5'-TACGCCGCAGTTCCTTACAAACTCTTCAC-3'[18] and DCX mRNA (Forward primer: 5'-CGCTATGCTCAGGATGATTTTTC-3'; reverse primer: 5'-GCTGTGGCTGATGGGTTTCC-3'; probe: 5'-CATGACTCGGCATTCATTTTCATCCAG-3') in a 7700 Sequence Detec-

\section{Bone Marrow}

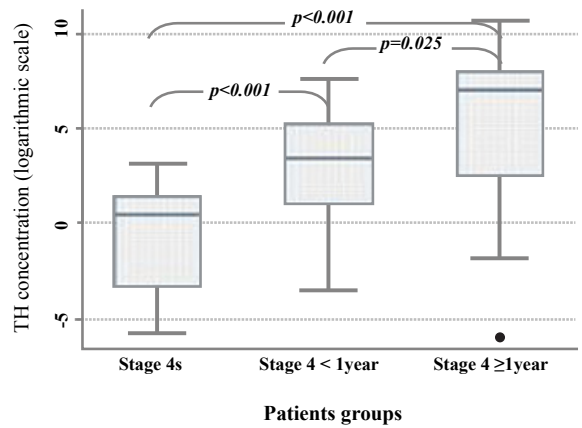

Peripheral Blood

$\mathrm{TH}$

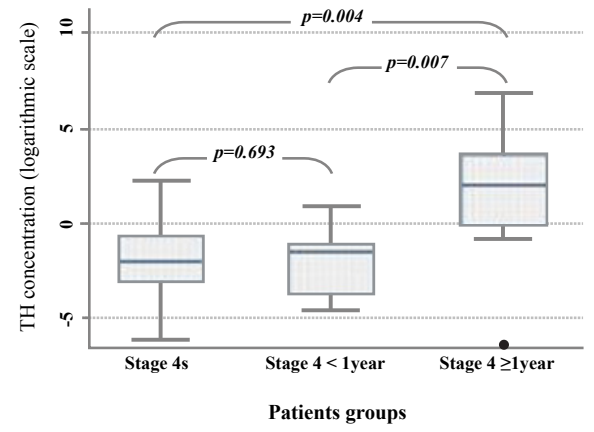

PHOX2B

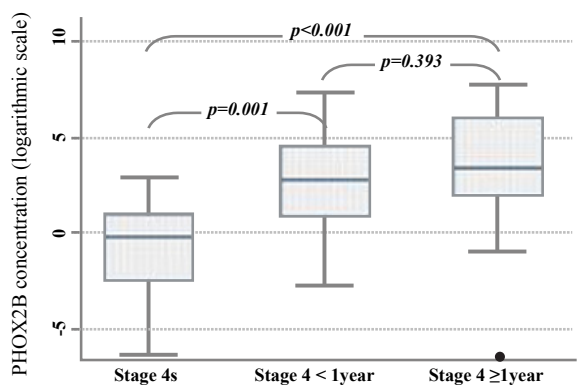

Patients groups

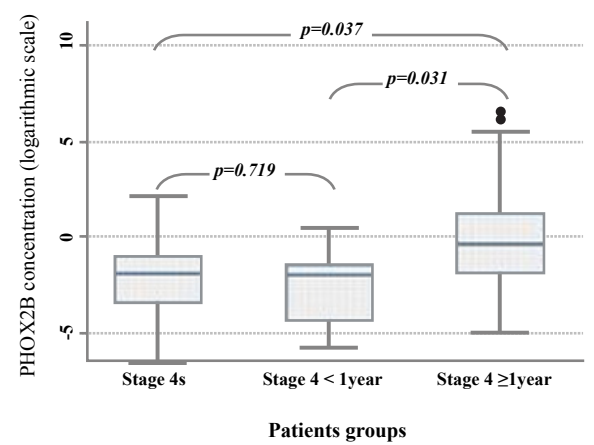

$D C X$
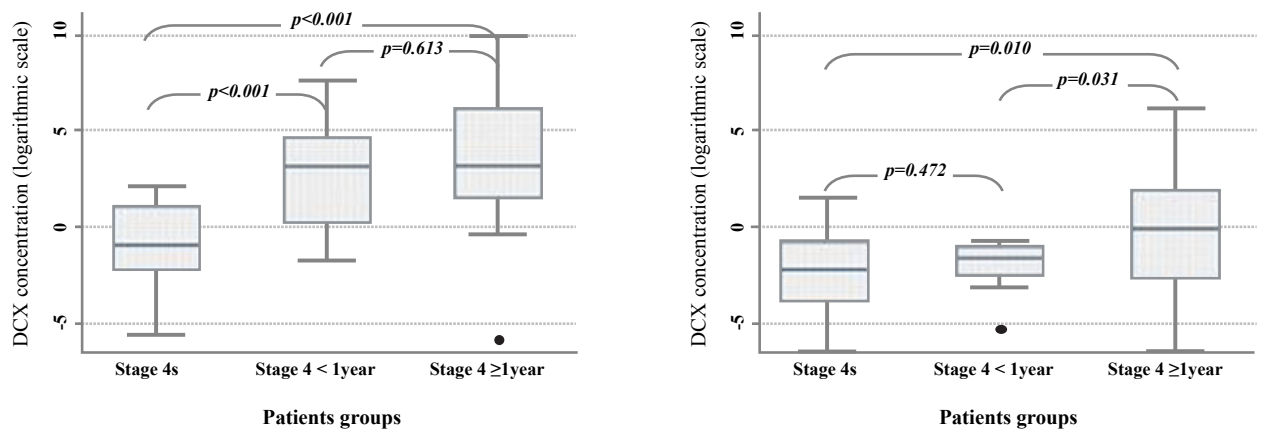

Figure 1: Level of expression of the three NB-related molecular markers in BM and PB samples from the three groups of study patients. 
tion System (Applied Biosystems, Foster City, CA). Negative controls (water as template and RNA reverse-transcribed without MMLV) and a positive control (cDNA from IMR-32 NB cell line) were run in each plate.

Samples were considered positive if at least two of the three quantification cycle $(\mathrm{Cq})$ values were lower than 40 . Positive results of RT-qPCR analysis were expressed as $\Delta \Delta$ Cq values [19] using $\beta 2$ - microglobulin as endogenous reference mRNA and the IMR-32 cell line as the exogenous reference sample, as described [14].

A checklist prepared according to the Minimum Information for Publication of Quantitative Real-Time PCR Experiments (MIQE) [20] is available in Doc S2.

\section{Statistical analysis}

Descriptive statistics were reported as percentages for categorical

\section{Bone Marrow}

A

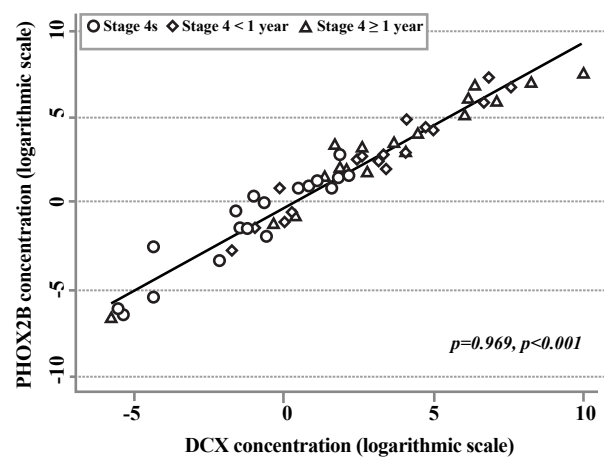

\section{Peripheral Blood}

\section{PHOX2B and DCX}

B

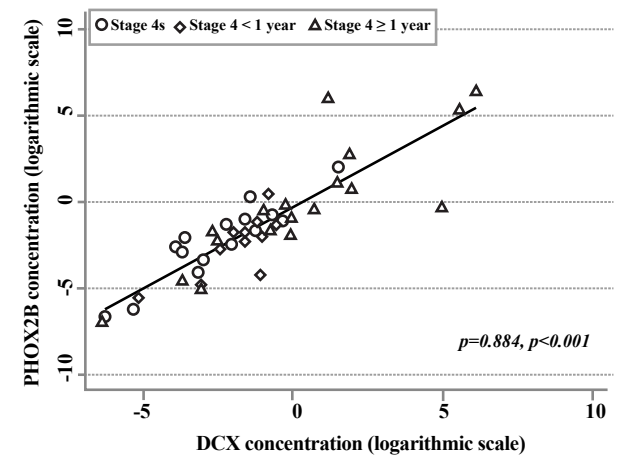

TH and PHOX2B

C

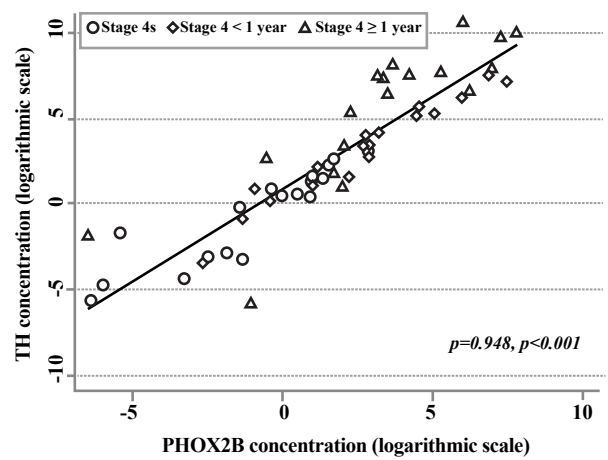

D

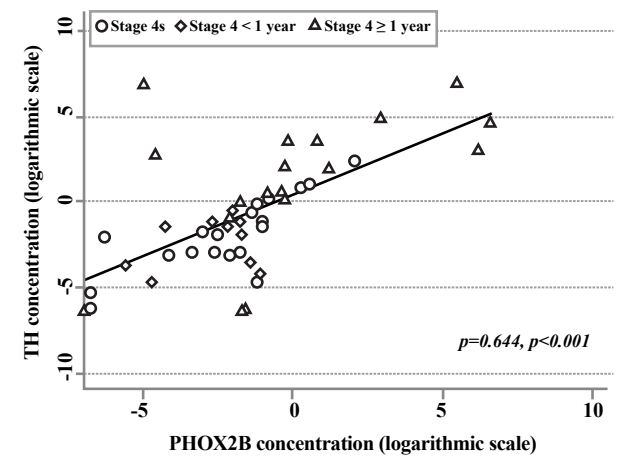

TH and DCX

E

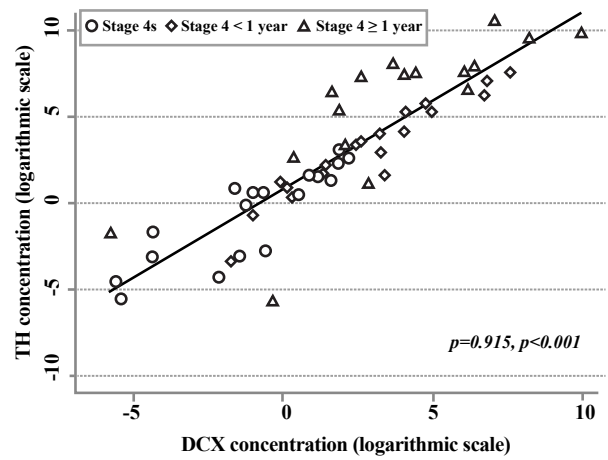

$\mathbf{F}$

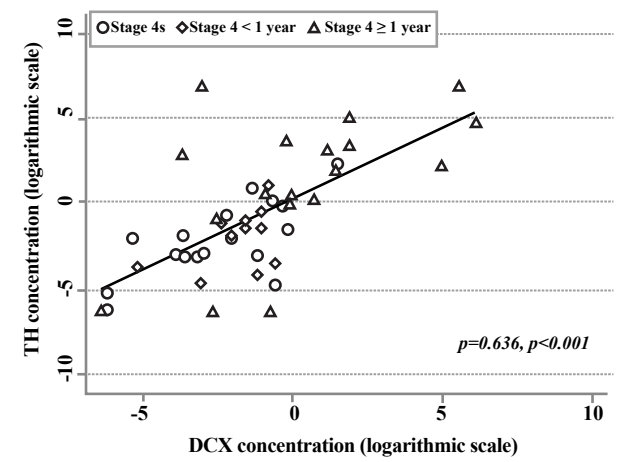

Figure 2: Correlation between $\triangle \Delta C q$ values measured with the three different markers in $B M$ (panels $A, C$ and $E$ ) and PB (panels $B, D$ and $F$ ) samples from the study patients. 
variables. For continuous data, medians with inter quartile range (IQR) were used due to the non normal distribution of the observations. Comparisons of frequency data were performed by means of the chi square test or the Fisher exact test, when appropriate, while differences in the distribution of continuous variables were analyzed by the Wilcoxon Mann-Whitney test. Correlation among concentrations of different molecular markers was assessed by the Spearman's coefficient.

The diagnostic performance of the molecular markers was evaluated by the Receiver Operating Characteristic (ROC) analysis [21]. Analyses were made using Stata for Windows statistical package (release 11.0, Stata Corporation, College Station, TX, USA).

\section{Results}

As shown in Table 1s, the three groups of patients with metastatic NB selected for the molecular analysis of their paired BM and PB samples were similar as for gender, primary site and, most importantly, for the percentage of patients with BM involvement evaluated by MIBG scintigraphy and BM smears. As expected genetic abnormalities, such as MYCN amplification and 1p deletion were more frequent in children than in infants.

When $\mathrm{PB}$ and $\mathrm{BM}$ samples collected at diagnosis from these patients were evaluated for TH, PHOX2B and DCX mRNA expression, the levels of the three markers progressively increased, starting from stage $4 S$ infants through stage 4 infants to stage 4 children (Figure 1), in both $\mathrm{BM}$ and $\mathrm{PB}$ samples. However, while in BM samples the levels were significantly different among the three clinical groups, in $\mathrm{PB}$ samples the expression levels were significantly different only between stage 4 children and infants, irrespective of stage 4 or 4 S (Figure 1). Similar results were obtained when the age cut-off was shifted from 12 to 18 months (data not shown).

A perfect correlation between $P H O X 2 B$ and $D C X$ results was found both in BM and $\mathrm{PB}$ samples, whereas correlations between $\mathrm{TH}$ and $P H O X 2 B$ and between $T H$ and $D C X$ were good in $\mathrm{BM}$, but intermediate in $\mathrm{PB}$ samples (Figure 2). When $\mathrm{BM}$ and $\mathrm{PB}$ samples from the same patient were considered, correlation between their expression levels was good for TH ( $\rho=0.763)$, but poor for PHOX2B and DCX ( $\rho=0.377$ and 0.393 , respectively, Figure $\mathrm{S1}$ ). By applying ROC analysis, BM TH $\Delta \Delta \mathrm{Cq}$ values were confirmed as the most accurate in discriminating the three groups of patients (Table 2s). A cut-off value of 19.8 allowed to allocate infants to stage 4 or 4 S, and a cut-off value of 738.6 allowed to allocate a patients to the correct age group with $78 \%$ accuracy.

We next evaluated whether TH, PHOX2B and DCX expression levels significantly associated with clinical features and/or with other known prognostic markers (Table S2). No significant correlation was found in both stage 4 and $4 \mathrm{~S}$ infants, whereas in stage 4 children BM expression values for any of the three markers, as well as PB TH values, significantly associated not only with the presence of BM infiltration, but also with skeletal metastasis ( $\mathrm{p}=0.008$ for $\mathrm{BM} T H$; $\mathrm{p}=0.032$ for $\mathrm{PB}$ $\mathrm{TH}, \mathrm{p}=0.009$ for BM PHOX2B and $\mathrm{p}=0.032$ for $\mathrm{BM} D C X)$.

\section{Discussion}

Age and presence of metastatic disease are major prognostic factors in NB, thus in metastatic patients the age at diagnosis greatly impacts on both prognosis and type of therapeutic intervention. Hereby, we showed that TH, PHOX $2 B$ and DCX expression levels measured at diagnosis in $\mathrm{BM}$ and $\mathrm{PB}$ samples from infants and children with stage 4 disease were significantly different. Interestingly, the expression levels in PB samples from stage 4 infants were very close to those measured in stage $4 \mathrm{~S}$ infants.

In general RT-qPCRs for $P H O X 2 B$ and $D C X$ gave very similar results, slightly different from those obtained for $T H$ expression, that seems to give more clinical information, as demonstrated in patients with localized disease [22].

While in infants the expression levels of NB-validated markers did not associate with any clinical and biological feature, in stage 4 children TH, PHOX2B and DCX expression levels in BM samples, as well as TH expression levels in $\mathrm{PB}$ samples, associated with presence of skeletal metastasis, suggesting that elevated levels may indicate a more aggressive behaviour of metastatic cells and confirming the best performance of TH over the other two markers. Since the study patients were highly representative of the whole cohort of metastatic NB patients, our data suggest that the levels of expression of validated markers [15] may associate to different outcomes. Future multicentre prospective studies, however, will be necessary to assess their individual/combined clinical significance, because the number of available samples in this singlecountry study did not allow to perform this type of analysis.

According to both INSS [1] and INRG-SS [2], BM infiltration must be evaluated by morphological examination of smears and trephine biopsies, and a cut off of $10 \%$ of BM-infiltrating neoplastic cells allocates infants to either stage 4 or 4 S. Since morphological analysis is highly dependent on the examiner's experience and skill, discrepancies in interpreting slides can not be excluded. It is interesting to note that, in spite of similar incidence of $\mathrm{NB}$ cases and identical frequency of all other NB stages, the percentages of stage 4 and $4 \mathrm{~S}$ infants are remarkably different between North America and Europe/Japan [2329]. Thus, the use of a quantitative method might also improve uniform staging across different countries.

In conclusion, RT-qPCR for validated markers should be compared with conventional morphological evaluation of BM infiltration and its potential clinical significance assessed in comparison to other prognostic markers evaluable in the primary tumors, such as favourable histology [7] and favourable genetic profiles [9-13].

\section{Acknowledgments}

The Authors wish to thank all the physicians and pathologists that collected and centralized the samples, and the parents and legal guardians that allowed the use of their children's samples for research studies. The excellent data management of Mrs Barbara Galleni is deeply acknowledged. The authors thank Mrs Anna Capurro for editing.

This study was supported by Fondazione Italiana per la Lotta al Neuroblastoma to MVC. BC and SP were recipients of Fondazione Italiana per la Lotta a Neuroblastoma fellowships.

\section{References}

1. Brodeur GM, Pritchard J, Berthold F, Carlsen NL, Castel V, et al. (1993) Revisions of the international criteria for neuroblastoma diagnosis staging and response to treatment. Prog Clin Biol Res11:1466-1477.

2. Cohn SL, Pearson AD, London WB, Monclair T, Ambros PF, et al. (2009) The International Neuroblastoma Risk Group (INRG) classification system: an INRG Task Force report. J Clin Oncol 27: 289-297.

3. London WB, Boni L, Simon T, Berthold F, Twist C, et al. (2005) The role of age in neuroblastoma risk stratification: the German, Italian, and children's oncology group perspectives. Cancer Lett 228: 257-266.

4. London WB, Castleberry RP, Matthay KK, Look AT, Seeger RC, et al. (2005) Evidence for an age cutoff greater than 365 days for neuroblastoma risk group stratification in the Children's Oncology Group J Clin Oncol 23: 6459-6465.

5. D'Angio GJ, Evans AE, Koop CE (1971) Special pattern of widespread neuroblastoma with a favourable prognosis. Lancet 1: 1046-1049. 
Citation: Parodi S, Erminio G, Carlini B, Garaventa A, D'Angelo P, et al. (2012) Infants and Children with Stage 4 Neuroblastoma Express Significantly Different Levels of Specific Molecular Markers. J Mol Biomark Diagn S2:008. doi:10.4172/2155-9929.S2-008

6. Benard J, Raguenez G, Kauffmann A, Valent A, Ripoche H, et al. (2008) MYCNnon-amplified metastatic neuroblastoma with good prognosis and spontaneous regression: a molecular portrait of stage 4S Mol Oncol 2: 261-271.

7. De Bernardi B, Gerrard M, Boni L, Rubie H, Canete A, et al. (2009) Excellent outcome with reduced treatment for infants with disseminated neuroblastoma without MYCN gene amplification. J Clin Oncol 27: 1034-1040.

8. Canete A, Gerrard M, Rubie H, Castel V, Di Cataldo A, et al. (2009) Poor survival for infants with MYCN-amplified metastatic neuroblastoma despite intensified treatment: the International Society of Paediatric Oncology European Neuroblastoma Experience J Clin Oncol 27: 1014-1019.

9. Spitz R, Oberthuer A, Zapatka M, Brors B, Hero B, et al. (2006) Oligonucleotide array-based comparative genomic hybridization $(\mathrm{aCGH})$ of 90 neuroblastomas reveals aberration patterns closely associated with relapse pattern and outcome Genes Chromosomes Cancer 45: 1130-1142.

10. Mosse YP, Diskin SJ, Wasserman N, Rinaldi K, Attiyeh EF, et al. (2007) Neuroblastomas have distinct genomic DNA profiles that predict clinical phenotype and regional gene expression. Genes Chromosomes Cancer 46: 936-949.

11. Schleiermacher G, Michon J, Ribeiro A, Pierron G, Mosseri V, et al. (2011) Segmental chromosomal alterations lead to a higher risk of relapse in infants with MYCN-non-amplified localised unresectable/disseminated neuroblastoma (a SIOPEN collaborative study). Br J Cancer 105:1940-1948.

12. Taggart DR, London WB, Schmidt ML, DuBois SG, Monclair TF, et al. (2011) Prognostic value of the stage 4S metastatic pattern and tumor biology in patients with metastatic neuroblastoma diagnosed between birth and 18 months of age. J Clin Oncol 29: 4358-4364.

13. Coco S, Theissen J, Scaruffi P, Stigliani S, Moretti T, et al. (2012) Agedependent accumulation of genomic aberrations and deregulation of cell cycle and telomerase genes in metastatic neuroblastoma. Int J Cancer.

14. Viprey V, Corrias M, Kagedal B, Oltra S, Swerts K, et al. (2007) Standardisation of operating procedures for the detection of minimal disease by QRT-PCR in children with neuroblastoma: quality assurance on behalf of SIOPEN-R-NET. Eur J Cancer 43: 341-350.

15. Viprey VF, Lastowska MA, Corrias MV, Swerts K, Jackson MS, et al. (2008) Minimal disease monitoring by QRT-PCR: guidelines for identification and systematic validation of molecular markers prior to evaluation in prospective clinical trials. J Pathol 216: 245-252.

16. Haupt R, Garaventa A, Gambini C, Parodi S, Cangemi G, et al. (2010) Improved survival of children with neuroblastoma between 1979 and 2005: a report of the Italian Neuroblastoma Registry. J Clin Oncol 28: 2331-2338.

17. Corrias MV, Haupt R, Carlini B, Parodi S, Rivabella L, et al. (2006) Peripheral blood stem cell tumor cell contamination and survival of neuroblastoma patients. Clin Cancer Res 12: 5680-5685.
18. Stutterheim J, Gerritsen A, Zappeij-Kannegieter L, Kleijn I, Dee R, et al. (2008) $\mathrm{PHOX} 2 \mathrm{~B}$ is a novel and specific marker for minimal residual disease testing in neuroblastoma. J Clin Oncol 26: 5443-5449.

19. Livak KJ, Schmittgen TD (2001) Analysis of relative gene expression data using real-time quantitative PCR and the 2(-Delta Delta C $(T)$ ) Method. Methods 25:402-408

20. Bustin SA, Benes V, Garson JA, Hellemans J, Huggett J, et al. (2009) The MIQE guidelines: minimum information for publication of quantitative real-time PCR experiments. Clin Chem 55: 611-622.

21. Hanley JA (1989) Receiver operating characteristic (ROC) methodology: the state of the art. Crit Rev Diagn Imaging 29: 307-335.

22. Corrias MV, Haupt R, Carlini B, Cappelli E, Giardino S, et al. (2012) Multiple target molecular monitoring of bone marrow and peripheral blood samples from patients with localized neuroblastoma and healthy donors. Pediatr Blood Cancer 58: 43-49.

23. Strother D, Shuster JJ, McWilliams N, Nitschke R, Smith El, et al. (1995) Results of pediatric oncology group protocol 8104 for infants with stages $D$ and DS neuroblastoma. J Pediatr Hematol Oncol 17: 254-259.

24. Katzenstein HM, Bowman LC, Brodeur GM, Thorner PS, Joshi VV, et al. (1998) Prognostic significance of age, MYCN oncogene amplification, tumor cell ploidy, and histology in 110 infants with stage $\mathrm{D}(\mathrm{S})$ neuroblastoma: the pediatric oncology group experience--a pediatric oncology group study. J Clin Oncol 16: 2007-2017.

25. Nickerson HJ, Matthay KK, Seeger RC, Brodeur GM, Shimada H, et al. (2000) Favorable biology and outcome of stage IV-S neuroblastoma with supportive care or minimal therapy: a Children's Cancer Group study. J Clin Oncol 18 : 477-486.

26. Schmidt ML, Lukens JN, Seeger RC, Brodeur GM, Shimada H, et al. (2000) Biologic factors determine prognosis in infants with stage IV neuroblastoma: A prospective Children's Cancer Group study. J Clin Oncol 18: 1260-1268.

27. Schleiermacher G, Rubie H, Hartmann O, Bergeron C, Chastagner P, et al. (2003) Treatment of stage 4s neuroblastoma--report of 10 years' experience of the French Society of Paediatric Oncology (SFOP). Br J Cancer 89: 470-476.

28. Di Cataldo A, Dau D, Conte M, Parodi S, De Bernardi B, et al. (2009) Diagnostic and prognostic markers in infants with disseminated neuroblastoma: a retrospective analysis from the Italian Cooperative Group for Neuroblastoma. Med Sci Monit 15: MT11-18.

29. Gigliotti AR, Di Cataldo A, Sorrentino S, Parodi S, Rizzo A, et al. (2009) Neuroblastoma in the newborn. A study of the Italian Neuroblastoma Registry. Eur J Cancer 45: 3220-3227

\footnotetext{
This article was originally published in a special issue, Cancer Biomarkers handled by Editor(s). Dr. Sudhir Srivastava, Cancer Biomarkers Research Group, National Institute of Health, USA; Dr. Shou-Jiang Gao, The University of Texas Health Science Centre at San Antonio, USA; Dr. Kenneth Maiese, University of Medicine \& Dentistry of New Jersey, New Jersey Medical School, USA
} 